\title{
The Asymptotic Behavior of Yang-Mills Fields in the Large
}

\author{
Vladimir Georgiev ${ }^{1}$ and Pedro Paulo Schirmer ${ }^{2 \star}$ \\ ${ }^{1}$ Institute of Mathematics, Bulgarian Academy of Sciences, Boul. Acad. Bonchev bl.8, Sofia 1113, \\ Bulgaria \\ ${ }^{2}$ Institut für Angewandte Mathematik der Universität Bonn, Wegelerstrasse 10, W-5300 Bonn 1, \\ Federal Republic of Germany
}

Received May 14, 1990; in revised form August 2, 1991

Abstract. We consider Yang-Mills fields in Minkowski space-time and prove that all spherically symmetric solutions in the canonical gauge decay in time, provided the initial data has finite conformal energy.

\section{Introduction}

We consider Yang-Mills equations in Minkowski Space-Time $R^{3+1}$ :

$$
\begin{aligned}
F_{\alpha \beta}^{; \beta} & =0, \\
{ }^{*} F_{\alpha \beta} ; \beta & =0 .
\end{aligned}
$$

$F_{A}: R^{3+1} \rightarrow \Lambda^{2} \mathscr{G}$ is the Yang-Mills curvature tensor of a Yang-Mills potential $A: R^{3+1} \rightarrow \Lambda^{1} \mathscr{G}$ and $\mathscr{G}$ is the Lie algebra of the gauge group $G$. System 1.1-1.2 is a non-linear hyperbolic system of partial differential equations after the choice of a gauge. The question of global existence has been settled already and one obtains global solutions in $H^{s}$ (cf. [4]) but no information about the asymptotic behavior of its solutions was obtained. It was proved later in [1] (see also [2]) the existence of global large solutions in the weighted Sobolev spaces $H^{s, \delta}$ together with the characterization of the asymptotic behavior in time. The major drawback though is the strong fall-off rate of the Cauchy data, requiring for example that the electric field decays like $E(0, x)=O\left(|x|^{-4}\right)$ as $|x| \rightarrow+\infty$. This excludes configurations containing Coulomb charges and also dipole-type waves. In the special case of small-amplitude solutions one can obtain estimates (cf. [3,11, 12 and 13]) which give the long-term behavior in time of the solutions.

The purpose of this work is to investigate solutions corresponding to dipoletype Cauchy data and to provide the time-asymptotics in the large amplitude sector. Our results apply to generic spherically symmetric Yang-Mills fields in the so-called canonical gauge ([12]):

$$
A_{0}=\sum_{l=1}^{N} \phi_{l}(t, r) \wp_{l},
$$

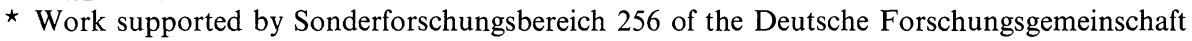




$$
\begin{aligned}
A_{i}= & \sum_{l=1}^{N} \psi_{l}(t, r) \wp_{l} \\
& +\frac{1}{r}\left[\sum_{l=1}^{N}\left(a_{1 l}\left[T_{m}, \wp_{l}\right] \varepsilon_{m j n} \frac{x_{n}}{r}+a_{2 l}\left[T_{j}, \wp_{l}\right]\right)-T_{j}\right] \varepsilon_{i j k} \frac{x_{k}}{r} .
\end{aligned}
$$

Here $a_{1 l}$ and $a_{2 l}$ are functions of $t, r$ alone, $T_{i}=\lambda_{*}\left(O_{(i)}\right)$ with $\lambda: S U(2) \rightarrow G$ a group homomorphism ${ }^{1}$ and $\wp_{l}$ is defined in terms of $s u(2)$ representation matrices:

$$
\wp_{j}=\sum_{m=-l_{j}}^{m=l_{j}} Y_{-m}^{l_{j}}\left(\frac{x}{r}\right) \mathscr{Y}_{m}^{l_{j}} .
$$

The functions $Y_{\underline{b}_{m}}^{l_{j}}$ are the standard spherical harmonic functions on the sphere and $\mathscr{Y}_{m}^{l}$ are a basis for an $s u(2)$ representation of dimension $2 l+1$ labeled by the third eigenvalue. ${ }^{2}$

The theorem proved here extends a result, due to Glassey and Strauss (reference [6]) concerning a particular class of $S U(2)$ spherically symmetric solutions, namely:

$$
\begin{aligned}
& A_{0}^{a}=0, \\
& A_{i}^{a}=\frac{\alpha(t, r)-1}{r} \varepsilon_{i a b} \frac{x_{b}}{r} .
\end{aligned}
$$

This is a special case of Ansatz 1.3-1.4 and the Yang-Mills equations reduce then to a single scalar wave equation. We shall extend this result for any group $G$ and when $F$ is of general spherically symmetric type. In this case the field equations cannot be significantly reduced and a more elaborate geometric analysis is needed. The goal of the work is to prove the following:

Theorem. Let $G$ be a compact semi-simple Lie group containing at least one $S U(2)-$ subgroup and let $(E(0), A(0))$ be initial data for the Yang-Mills equations 1.1-1.2 satisfying the constraint equation $\operatorname{Div}_{A} E=0$ and the spherically symmetric Ansatz 1.3-1.4. Assume that the conformal energy

$$
E_{0}=\int_{R^{3}}\left(1+|x|^{2}\right)|F(0, x)|^{2} d x
$$

is finite $E_{0}<+\infty$ and the initial data satisfy the estimate

$$
|F(0, \cdot)|_{1}:=\sup _{|\alpha| \leqq 1}\left(\sup _{x}(1+|x|)^{5 / 2+|\alpha|}\left|\partial_{x}^{\alpha} F(0, x)\right|\right)<+\infty .
$$

It follows that the solution exists globally and satisfies the decay rate

$$
|F(t, \cdot)|_{\infty} \leqq C_{0}(1+t)^{-1}
$$

with $C_{0}$ depending only on the conformal energy $E_{0}$ and the norms $|F(0, \cdot)|_{1}$.

\footnotetext{
${ }^{1}$ One proves that all possible lifts of the action of the rotation group on the base space $R^{3}$ to the total space of the $G$-bundle will be in one to one correspondence with homomorphisms $\lambda: S U(2) \rightarrow G$, when $G$ contains at least one $S U(2)$-subgroup. One says then that this mapping determines the type of spherical symmetry of the gauge field $F_{A}$. See [12] and references therein 2 cf. [8] for more details
} 


\section{Remarks.}

1. The constraint equation $\operatorname{Div}_{A} E=0$ is preserved by the non-linear flow and it will be automatically verified for later times.

2. The global existence part of the theorem follows from [4]. One has only to remark that the Yang-Mills flow preserves the canonical class of potentials 1.3-1.4 (cf. [12]).

3. Due to the asymptotic behavior of the initial data, configurations containing Coulomb charges cannot still be accommodated in the hypotheses of this theorem. In that case the fall-off rate of the electric field is of order $O\left(r^{-2}\right)$.

The proof of this theorem consists of a non-trivial bootstrapping argument. To clarify the proof we present here the main ideas. The basic notation and the local energy estimates are presented in Sect. 2.

The decay estimates are obtained by a decomposition of Minkowski space-time into two different regions: a small cone of aperture $\varepsilon>0$ around the central line, which we call the interior region $\Sigma_{i}$, and its complement, $\Sigma_{e}$, named by us the exterior region. The number $\varepsilon$ depends only on the initial data as $E_{0}^{-1}$, so that in the case the initial data has very large energy our interior region will reduce to a very narrow cone. The plan is the following: On Sect. 4 and its subsections we use the spherically symmetric Ansatz and apply the method of integration along characteristics. We are able to prove an estimate of the kind

$$
Q(t) \leqq C_{0}+c[F(t)]_{\infty},
$$

where $Q(t)$ and $[F(t)]_{\infty}$ denote the norms ${ }^{3}$

$$
\begin{gathered}
{[F(t)]_{\infty}=\sup _{\Sigma_{i}(t)}(1+|x|)(1+\tau+|x|)|F(\tau, x)|} \\
Q(t)=\sup _{\Sigma_{e}(t)}\left(|x|^{2}|\rho(t, x)|+|x|^{2}|\sigma(t, x)|+(1+t+|x|)|x|^{3 / 2}|\alpha(t, x)|\right. \\
+|x|(1+|t-| x||)|\underline{\alpha}(t, x)|) .
\end{gathered}
$$

Here $\rho, \sigma, \alpha$ and $\underline{\alpha}$ are the null components of $F$ relative to a null frame (cf. Sect. 2). This decomposition is the key to exploit (in the wave zone) an appropriate version of the null condition of Klainerman.

The last term on the right-hand side of 1.10 is due to the fact that for one component of $F$ (namely the component $\underline{\alpha}$ ) one can only integrate along the outgoing direction. Here we integrate towards the central line, up to the boundary of our interior region, where the field can be estimated in a different way. The exterior estimates are independent of the choice of a gauge but rely heavily on the spherically symmetric Ansatz. The second part (Sect. 5) concerns the interior region. We follow the approach of [4] and use a local gauge here. By using the fundamental solution representation of the wave operator we are able to obtain an estimate of the type

$$
[F(t)]_{\infty} \leqq C+C_{0} \varepsilon[F(t)]_{\infty} .
$$

By choosing $\varepsilon$ small enough we are able to close the argument.

\footnotetext{
${ }^{3}$ Here we define $\Sigma_{i}(t)=\Sigma_{i} \cap\{s \leqq t\}$ and $\Sigma_{e}(t)=\Sigma_{e} \cap\{s \leqq t\}$
} 


\section{Notation}

In this section we record the definitions used in the course of the paper. We shall consider Minkowski space-time $R^{3+1}$ with coordinates $(t, x)=\left(x^{0}, \ldots, x^{3}\right)$ and endowed with the flat metric $\eta=-d t^{2}+d r^{2}+r^{2} d \Omega_{S^{2}}$. We also use Einstein's convention of raising and lowering indices. The gauge group is a Lie group $G$ and we denote its Lie algebra by $\mathscr{G}$ and the Lie algebra commutator by $[.,$.$] . The gauge$ group $G$ is assumed to be compact and semi-simple. In particular, the Lie algebra $\mathscr{G}$ admits a Killing form, namely a bilinear symmetric positive definite form that is invariant under the Ad action. In the sequel we will often write '. ' for this bilinear form. Finally, we fix a basis $T_{a}, a=1,2, \ldots, N$ of $\mathscr{G}$ which is orthonormal with respect the Killing form. The Yang-Mills potential is a $\mathscr{G}$-valued 1 -form $A=A_{\mu} d x^{\mu}=\left(A_{\mu}^{a} T_{a}\right) d x^{\mu}$. The Yang-Mills field-strength of $A$ is a 2-form $F_{A}: R^{3+1} \rightarrow \Lambda^{2} \mathscr{G}$ defined as $F_{\mu \nu}=\partial_{\mu} A_{v}-\partial_{v} A_{\mu}+\left[A_{\mu}, A_{v}\right] . \quad D$ will denote the covariant derivative $D_{\mu}=\partial_{\mu}+\left[A_{\mu}, \cdot\right]$. We will also refer to it by the use of semicolon ";". The gauge copies of $A$ are denoted ${ }^{(g)} A=g A g^{-1}-d g g^{-1}$, where $g: R^{3+1} \rightarrow G$ takes values in the group $G$. The corresponding curvature tensor and covariant derivative will change accordingly as ${ }^{(g)} F=g F g^{-1}$ and ${ }^{(g)} D^{(g)} F=g D F g^{-1}$.

We shall introduce the null frame

$$
\left\{e_{1}, e_{2}, e_{3}, e_{4}\right\},
$$

where $e_{3}=\partial_{t}-\partial_{r}, e_{4}=\partial_{t}+\partial_{r}$ and $e_{A}=\frac{1}{r} \xi_{A}, \xi_{A}$ the standard orthonormal frame tangent to the unit sphere. Associated with $\xi_{A}$ we consider the angular gradient $\nabla$ and the angular operators $\nabla \cdot u=\bigotimes_{A} u_{A}, \not \times u=\varepsilon_{A B}\left(\nabla_{A} u_{B}-\nabla_{B} u_{A}\right)$ defined for every 1 -form $u$ tangent to the spheres $r=$ constant.

In connection with 2.1 we shall use the weights

$$
\tau_{-}=1+|\tau-r|, \quad \tau_{+}=1+\tau+r
$$

and define the null decomposition of $F$ as follows:

$$
\begin{aligned}
\underline{\alpha}_{A} & =F_{A 3}, & \alpha_{A} & =F_{A 4}, \\
\rho & =\frac{1}{2} F_{34}, & \sigma & =F_{12} .
\end{aligned}
$$

We will also need in the sequel a localized null decomposition of the curvature tensor $F$. Denote by $K_{p}$ the backward light-cone of an arbitrary point $p=\left(t_{0}, x_{0}\right)$ :

$$
K_{p}=\left\{(t, x)\left|t-t_{0}=\right| x-x_{0} \mid\right\}
$$

and by $B_{p}$ its intersection with the initial data hyperplane $t=0$. Introduce local coordinates $r=t-t_{0}, \omega=\frac{x-x_{0}}{\left|x-x_{0}\right|}$, so that we can write an arbitrary point in $K_{p}$ as

$$
t=t_{0}+r, \quad x=x_{0}+r \omega .
$$

We define the local null frame

$$
\left\{{ }^{(p)} e_{1},{ }^{(p)} e_{2},{ }^{(p)} e_{3},{ }^{(p)} e_{4}\right\}
$$


relative to the vertex $p$ as follows. The local null vectors are

$$
{ }^{(p)} e_{3}=\partial_{t}-\frac{x^{i}-x_{0}^{i}}{\left|x-x_{0}\right|} \partial_{i}, \quad{ }^{(p)} e_{4}=\partial_{t}+\frac{x^{i}-x_{0}^{i}}{\left|x-x_{0}\right|} \partial_{i}
$$

The tangent frame is ${ }^{(p)} e_{A}=\frac{1}{r}{ }^{(p)} \xi_{A}$, where ${ }^{(p)} \xi_{A}$ denotes the standard local orthonormal frame tangent to the unit sphere with center $x_{0}$. We will also use the local light-cone coordinates

$$
{ }^{(p)} x^{3}=\frac{\tau-r}{2}, \quad{ }^{(p)} x^{4}=\frac{\tau+r}{2},
$$

and the local null decomposition

$$
\begin{aligned}
{ }^{(p)} \underline{\alpha}_{A} & =F_{A 3}, \quad{ }^{(p)} \alpha_{A}=F_{A 4}, \\
{ }^{(p)} \rho & =1 / 2 F_{34}, \quad{ }^{(p)} \sigma=F_{12} .
\end{aligned}
$$

When there is no risk of confusion we shall drop the superscripts $(p)$.

\section{Energy Estimates}

In this section we shall recall some basic facts concerning the energy identities of the Yang-Mills field. We also consider a local energy estimate which is used together with the fundamental solution representation of the wave equation.

The energy-momentum tensor of the Yang-Mills field $F$ is defined as

$$
Q_{\mu \nu}=F_{\mu}^{\alpha} \cdot F_{\alpha \nu}-\frac{1}{4} \eta_{\mu \nu} F_{\xi \lambda} \cdot F^{\xi \lambda}
$$

If $X, Y$ are vector fields in Minkowski space we write $Q(X, Y)=Q_{\mu \nu} X^{\mu} Y^{\nu}$. It is known ${ }^{4}$ that the tensor $Q$ is a symmetric traceless 2-tensor and that it satisfies the positivity condition $Q(X, Y) \geqq 0$ for any pair of non space-like future-directed vectors $X$ and $Y$. Computing its divergence we find

$$
T_{\mu v}{ }^{, v}=0 \text {. }
$$

Now, if $X$ is a conformally Killing vector field, i.e., $X^{\mu, v}+X^{v, \mu}=\Lambda \eta^{\mu v}$, for some function $\Lambda$, then defining the 4-momentum vector $P_{\mu}=Q_{\mu \nu} X^{\nu}$ we obtain

$$
\partial_{\mu} P^{\mu}=0 \text {. }
$$

We can now integrate this equation on a space-time domain $\mathscr{D}$ bounded by two space-like hypersurfaces $\Sigma_{0}, \Sigma_{1}$ and by a null cone $C$. Letting $B_{i}, i=1,2$ denote the intersections of $\Sigma_{i}$ and $C, i=1,2$ and $N$ denote the normal unit vector to $\Sigma$ we obtain

$$
\int_{B_{1}}\langle P, N\rangle=\int_{B_{0}}\langle P, N\rangle+\int_{C}\left\langle P, e_{3}\right\rangle
$$

\footnotetext{
${ }^{4}$ cf. [7] for example
} 
in the case $C$ is a backward light-cone and

$$
\int_{B_{1}}\langle P, N\rangle=\int_{B_{0}}\langle P, N\rangle-\int_{C}\left\langle P, e_{4}\right\rangle
$$

in the case $C$ is a forward light-cone.

We shall consider the case when one of the hypersurfaces reduces to a point $p$ and $C$ is either the forward or backward light-cone $K_{p}$ of the point $p$. In this case $N=T=\partial_{t}$ and we choose $X$ to be the conformal vector field

$$
X=K=\left(1+t^{2}+r^{2}\right) \frac{\partial}{\partial t}+2 t x^{i} \frac{\partial}{\partial x^{i}} .
$$

Equations 3.3-3.4 give

$$
\int_{K_{p}} Q\left({ }^{(p)} e_{4}, K\right) d \Sigma_{q}=\int_{\tau=t} Q(T, K) d x \leqq E_{0}(t)
$$

in the case $K_{p}$ is the forward light-cone of $p$ and

$$
\int_{K_{p}} Q\left({ }^{(p)} e_{3}, K\right) d \Sigma_{q}=\int_{\tau=t} Q(T, K) d x \leqq E_{0}(t)
$$

in the case $K_{p}$ is the backward light-cone of $p$. Here $E_{0}$ is the total conformal energy of the Yang-Mills field at the time $t$ :

$$
E_{0}(t)=\int_{\tau=t}\left(1+t^{2}+r^{2}\right)\left(|F|^{2}+|* F|^{2}\right) d x .
$$

We remark that $E_{0}(t)=E_{0}$ is a conserved quantity, as it can be seen from integrating Eq. 3.2 in a slab $[0, t] \times R^{3}$. Our next result consists of using the energy estimates to obtain some $L^{2}$-bounds of the null decomposition of the tensor $F$. We first consider the decomposition relative to the origin:

Proposition 3.1. If the Yang-Mills curvature tensor F satisfies Eqs. 1.1-1.2, and 2.2-2.3 denote its global null decomposition, then the following estimates are verified

$$
\int_{K_{p}} \tau_{-}^{2}|\underline{\alpha}|^{2}+\tau_{+}^{2}\left(|\rho|^{2}+|\sigma|^{2}\right) d \Sigma_{q} \leqq c E_{0}
$$

for every backward light-cone $K_{p}$ with vertex $p$ on the central line $r=0$. Also

$$
\int_{K_{p}} \tau_{+}^{2}|\alpha|^{2}+\tau_{-}^{2}\left(|\rho|^{2}+|\sigma|^{2}\right) d \Sigma_{q} \leqq c E_{0}
$$

for every advanced light-cone $K_{p}$ with vertex $p$ on the central line $r=0$.

The proof of this proposition consist of computing the energy-momentum tensor in null coordinates:

$$
\begin{aligned}
& T\left(e_{3}, K\right)=\tau_{-}^{2} T_{33}+\tau_{+}^{2} T_{34}, \\
& T\left(e_{4}, K\right)=\tau_{-}^{2} T_{34}+\tau_{+}^{2} T_{44},
\end{aligned}
$$

where

$$
T_{33}=|\underline{\alpha}|^{2}, \quad T_{34}=|\rho|^{2}+|\sigma|^{2}, \quad T_{44}=|\alpha|^{2} .
$$

These energy bounds are sufficient in the exterior region. To estimate the field in the interior region we need to estimate the energy on a cone with vertex $p$ not necessarily on $r=0$. We are able to prove anyhow the following: 
Proposition 3.2. If the Yang-Mills curvature tensor $F$ satisfies Eqs. 1.1-1.2, and 2.6-2.7 denote its local null decomposition relative to the point $p$, then for the backward light-cone $K_{p}$ with vertex $p$ the following estimate is verified:

$$
\int_{K_{p}} \tau_{-}^{2}(q)\left(|\underline{\alpha}|^{2}+|\rho|^{2}+|\sigma|^{2}\right) d \Sigma_{q} \leqq c E_{0} .
$$

The proof is similar to the previous one, with a few technical details added. In local null coordinates we have

$$
\begin{aligned}
& T_{34}=|\rho|^{2}+|\sigma|^{2}, \\
& T_{33}=|\underline{\alpha}|^{2}, \\
& T_{44}=|\alpha|^{2}, \\
& T_{3 A}=-\rho \cdot \underline{\alpha}_{A}+\underline{\alpha}_{B} \varepsilon_{B A} \sigma, \\
& T_{4 A}=-\rho \cdot \alpha_{A}+\alpha_{B} \varepsilon_{B A} \sigma .
\end{aligned}
$$

Computing further in local coordinates:

$$
T\left({ }^{(p)} e_{3}, K\right)=K^{3}|\underline{\alpha}|^{2}+K^{A}\left(-\rho \cdot \underline{\alpha}_{A}+\underline{\alpha}_{B} \varepsilon_{B A} \sigma\right)+K^{4}\left(\rho^{2}+\sigma^{2}\right) .
$$

Introducing the vector $\mathscr{U}=\left(\underline{\alpha}_{1}, \underline{\alpha}_{2}, \rho, \sigma\right)$ in the $4 N$-dimensional space $\underbrace{\mathscr{G} \times \ldots \times \mathscr{G}}_{4}$ we can represent the last equation in terms of a quadractic form $B:^{5}$

$$
T\left({ }^{(p)} e_{3}, K\right)=\langle\mathscr{U}, B \mathscr{U}\rangle,
$$

where $\langle\mathscr{U}, \mathscr{V}\rangle=\sum_{i} \mathscr{U}_{i} \cdot \mathscr{V}_{i}$ and $B$ denotes the $4 N \times 4 N$ symmetric matrix

$$
B=\left(\begin{array}{cccc}
K^{3} & 0 & -\frac{1}{2} K^{1} & -\frac{1}{2} K^{2} \\
0 & K^{3} & -\frac{1}{2} K^{2} & -\frac{1}{2} K^{1} \\
-\frac{1}{2} K^{1} & -\frac{1}{2} K^{2} & K^{4} & 0 \\
\frac{1}{2} K^{2} & -\frac{1}{2} K^{1} & 0 & K^{4}
\end{array}\right)
$$

The form $B$ can be diagonalized by means of a unitary matrix $P$, i.e., $B=P^{-1} \Lambda P$, with $\Lambda=\operatorname{diag}\left(\lambda_{1}, \ldots, \lambda_{4}\right)$, so that by setting $\mathscr{V}=P \mathscr{U}$ and $m=\inf _{i} \lambda_{i}$ we get

$$
\begin{aligned}
T\left({ }^{(p)} e_{3}, K\right) & =\langle\mathscr{V}, \Lambda \mathscr{V}\rangle=\sum_{i}\left|\mathscr{V}_{i}\right|^{2} \lambda_{i} \\
& \geqq m|\mathscr{V}|^{2}=m|\mathscr{U}|^{2}=m\left(|\underline{\alpha}|^{2}+\rho^{2}+\sigma^{2}\right) .
\end{aligned}
$$

The infimum $m$ is computed by solving the eigenvalue equation $\operatorname{det}(B-\lambda I)=0$ and estimating from below the eigenvalues. One finds

$$
\left(\lambda^{2}-\lambda\left(K^{3}+K^{4}\right)+\left(K^{3} K^{4}-\frac{1}{4}\left(\left(K^{1}\right)^{2}+\left(K^{2}\right)^{2}\right)\right)\right)^{2}=0 .
$$

The linear term in $\lambda$ is just $2 K^{0}=2\left(1+t^{2}+|x|^{2}\right)$ while the last term equals $-\frac{1}{4} K^{2}, K^{2}=-\tau_{-}^{2} \tau_{+}^{2}$. From this it will follow that $m \geqq \frac{1}{8} \tau_{-}^{2}$ and the proposition will follow.

\footnotetext{
${ }^{5}$ We shall drop here the dependence on the Lie algebra parameters
} 


\section{Estimates of the Solution for $|x|+1 \geqq \varepsilon t$}

Let $0<\varepsilon<1$ be a fixed real number. In this section we shall estimate the curvature tensor $F(t, x)$ in the exterior domain:

$$
1+|x| \geqq \varepsilon t
$$

The exterior region includes the wave-zone $|x|+1=t$, along which the curvature tensor cannot decay better than $t^{-1}$. There we must take advantage of the asymptotic behavior of the components of $F$ to exploit the null condition in the non-linearities. For that matter we shall use the representation of the Yang-Mills equations in light-cone coordinates (see [12]):

$$
\begin{array}{r}
\left(D_{4}+\frac{1}{r}\right) \underline{\alpha}_{A}+D_{A} \rho-\varepsilon_{A B} D_{B} \sigma=0, \\
\left(D_{3}-\frac{1}{r}\right) \alpha_{A}-D_{A} \rho-\varepsilon_{A B} \not D_{B} \sigma=0, \\
\left(D_{4}+\frac{2}{r}\right) \rho+\not D \cdot \alpha=0, \\
\left(D_{3}-\frac{2}{r}\right) \rho-\not D \cdot \underline{\alpha}=0, \\
\left(D_{4}+\frac{2}{r}\right) \sigma+\not D \times \alpha=0, \\
\left(D_{3}-\frac{2}{r}\right) \sigma+\not D \times \underline{\alpha}=0 .
\end{array}
$$

Using now the relation

$$
F \cdot D_{\lambda} F+D_{\lambda} F \cdot F=\partial_{\lambda}(F \cdot F),
$$

we obtain from $4.2-4.7$ the following equations:

$$
\begin{aligned}
\partial_{4}\left(\left|r \underline{\alpha}_{A}\right|^{2}\right) & =-r^{2} \underline{\alpha}_{A} \cdot\left(\not D_{A} \rho-\varepsilon_{A B} \not_{B} \sigma\right), \\
\partial_{3}\left(\left|r \alpha_{A}\right|^{2}\right) & =r^{2} \alpha_{A} \cdot\left(\not D_{A} \rho-\varepsilon_{A B} \not_{B} \sigma\right), \\
\partial_{4}\left(r^{4} \rho^{2}\right) & =-r^{4} \not D \alpha \cdot \rho \\
\partial_{3}\left(r^{4} \rho^{2}\right) & =r^{4} \not D \underline{\alpha} \cdot \rho \\
\partial_{4}\left(r^{4} \sigma^{2}\right) & =-r^{4}(\not D \times \alpha) \cdot \sigma \\
\partial_{3}\left(r^{4} \sigma^{2}\right) & =r^{4}(\not D \times \underline{\alpha}) \cdot \sigma .
\end{aligned}
$$

The terms appearing on the right-hand sides of Eqs. 4.9-4.14 can be estimated by using the spherically symmetric Ansatz 1.3-1.4. The invariant connections can be characterized infinitesimally by

$$
\mathscr{L}_{O_{(i)}} F+\left[T_{i}, F\right]=0
$$


for all $i=1,2,3$, where $T_{i}=\lambda_{*}\left(O_{(i)}\right)$ denote the matrices appearing in the definition $1.3-1.4$ of the spherically symmetric Ansatz. ${ }^{6}$

Our task now consists of estimating the right-hand-sides of Eqs. 4.9-4.14 by lower order terms. This is essentially achieved using Eq. 4.15.

Proposition 4.1. Let $F_{A}: R^{3+1} \rightarrow \Lambda^{2} \mathscr{G}$ a spherically symmetric tensor. It follows that in the exterior region we have

$$
\left|r D_{A} \omega_{B}\right| \leqq c\left(1+\left|r^{2} \sigma\right|_{\infty, \mathrm{ext}}^{1 / 2}\right)|\omega|
$$

for every component $\omega_{B}$ of $F$ relative to the null frame and every derivative $\nabla_{A}$.

Proof. Breaking up the covariant derivative and the gauge-covariant derivative we have

$$
\begin{aligned}
\not_{A} \omega_{B} & =D_{A} \omega_{B}+\text { lower order terms } \\
& =\nexists_{A} \omega_{B}+\left[A_{A}, \omega_{B}\right]+1 . \text { o.t. }
\end{aligned}
$$

The lower order terms are directly estimated by $r^{-1}|\omega|$, while the others are treated as follows. Applying the Lie derivatives $\mathscr{L}_{\boldsymbol{O}_{(i)}}$ we obtain for an arbitrary component $\omega_{B}$ :

$$
\left|r \phi_{A} \omega_{B}\right|^{2} \leqq \sum_{i=1}^{3}\left|\mathscr{L}_{O_{(i)}} \omega_{B}\right|^{2}=\sum_{i=1}^{3}\left|-\left[T_{i}, \omega_{B}\right]\right|^{2} \leqq c|\omega|^{2} .
$$

The last term requires a more subtle argument. To control the tangential components $A$ of the potential we look back to the Ansatz 1.3-1.4 and observe that the normal component $\sigma$ of the curvature is completely constrained by the tangential components of the potential. Indeed, computing the magnetic curvature:

$$
\sigma=\frac{1}{r^{2}} \sum_{l=1}^{N}\left(a_{1 l}^{2}+a_{2 l}^{2}-1\right) \wp_{l} .
$$

This completes the proof of the proposition.

With this estimate one can prove the following result:

Proposition 4.2. There exists a constant $C=c E_{0}^{1 / 4}\left(1+c E_{0}^{1 / 2}|F(0)|_{1}\right)^{1 / 2}+|F(0)|_{1}$ depending only on the initial data such that the following estimates for the null components $\left\{\underline{\alpha}_{A}, \alpha_{A}, \rho, \sigma\right\}$ of the Yang-Mills curvature tensor $F$ are verified for all points $(t, x)$ in the exterior region $|x| \geqq 1,|x|+1 \geqq \varepsilon t$ :

$$
\begin{aligned}
& |\rho(t, x)| \leqq C|x|^{-2}, \\
& |\sigma(t, x)| \leqq C|x|^{-2}, \\
& |\alpha(t, x)| \leqq C(1+t+|x|)^{-1}|x|^{-3 / 2}, \\
& |\underline{\alpha}(t, x)| \leqq C|x|^{-1}(1+|t-| x||)^{-1}\left(1+[F(t)]_{\infty}\right),
\end{aligned}
$$

\footnotetext{
${ }^{6}$ They measure the isospin contribution to the total angular momentum whereas $\mathscr{L}_{\boldsymbol{O}_{\text {(i) }}}$ measures the orbital angular momentum. The operators $\hat{\mathscr{L}}_{O_{(i)}}=\mathscr{L}_{O_{(i)}}+\left[T_{i}, \cdot\right]$ satisfy the commutation rules $\left[\hat{\mathscr{L}}_{o_{(i)}}, \hat{\mathscr{L}}_{O_{(j)}}\right]-\varepsilon_{i j k} \hat{\mathscr{L}}_{O_{(k)}}=0$ when applied to the spherically symmetric tensors $1.3-1.4$
} 
where

$$
[F(t)]_{\infty}=\sup _{\Sigma_{i}(t)}(1+|x|)(1+\tau+|x|)|F(\tau, x)|
$$

measures the curvature tensor $F$ in the interior region $\Sigma_{i}(t): 1+|x| \leqq \varepsilon \tau, \tau \leqq t$.

The estimates of the curvature will be established in the next subsections. This will be done in three separate steps.

4.1. Estimates of the Components $\rho$ and $\sigma$. First we shall estimate $\rho$ and $\sigma$. Set

$$
Q(t)=\sup _{\Sigma_{e}(t)}\left(r^{2}|\rho(\tau, x)|+r^{2}|\sigma(\tau, x)|\right),
$$

where $\Sigma_{e}(t)$ denotes the intersection of the exterior region $|x|+1 \geqq \varepsilon \tau$ with the region $\{\tau \leqq t\}$.

Let now $\left(t_{0}, x_{0}\right)$ belong to the exterior domain. Integrating Eq. 4.12 along the characteristic $v=t+r=$ constant we get

$$
r_{0}^{4}\left|\rho\left(t_{0}, x_{0}\right)\right|^{2} \leqq Q(0)^{2}+c \int_{u_{*}}^{u_{0}} r^{4}\left|\rho\left(u, v_{0}\right)\right| \cdot|\not \underline{\alpha}| d u
$$

where

$$
r_{0}=\left|x_{0}\right|, \quad u_{0}=t_{0}-\left|x_{0}\right|, \quad v_{0}=t_{0}+\left|x_{0}\right|, \quad u_{*}=-t_{0}-\left|x_{0}\right| .
$$

Using 4.16 and the Cauchy-Schwarz inequality, we obtain

$$
\begin{aligned}
& \int_{u_{*}}^{u_{0}} r^{4}\left|\rho\left(u, v_{0}\right)\right| \cdot|\not \underline{\alpha}| d u \leqq c \int_{u_{*}}^{u_{0}} r \cdot|\underline{\alpha}| r^{2}|\rho| \cdot\left(1+\left|r^{2} \sigma\right|^{1 / 2}\right) d u \\
& \leqq c\left(\int_{u_{*}}^{u_{0}} r^{2}|\underline{\alpha}|^{2}(1+u)^{2} d u\right)^{1 / 2} \\
& \cdot\left(\int_{u_{*}}^{u_{0}} r^{4}|\rho|^{2}(1+u)^{-2}\left(1+\left|r^{2} \sigma\right|\right) d u\right)^{1 / 2} .
\end{aligned}
$$

The first factor can be bounded now by looking to the local energy of the field $F$ in the backward light-cones. One considers the backward cone $K_{p}$ with vertex $p=\left(v_{0}, 0,0,0\right)$ and applies Proposition 3.1. We remark that the surface measure is $d \Sigma_{p}=r^{2} d u d \omega$ and that for this particular cone $\tau_{-}^{2}=(1+u)^{2}$. We get then

$$
\begin{aligned}
\int_{u_{*}}^{u_{0}} r^{4}\left|\rho\left(u, v_{0}\right)\right| \cdot|\not \underline{\alpha}| d u & \leqq c E_{0}^{1 / 2}\left(Q+Q^{3 / 2}\right) \cdot\left(\int_{u_{*}}^{u_{0}}(1+u)^{-2} d u\right)^{1 / 2} \\
& \leqq c E_{0}^{1 / 2}\left(Q+Q^{3 / 2}\right),
\end{aligned}
$$

where $E_{0}$ is the conformal energy of $F$. Henceforth

$$
\left|r_{0}^{2} \rho\right|^{2} \leqq Q(0)^{2}+c E_{0}^{1 / 2}\left(Q\left(t_{0}\right)+Q\left(t_{0}\right)^{3 / 2}\right) .
$$

Similarly, using now Eq. 4.14 we get

$$
\left|r_{0}^{2} \sigma\right|^{2} \leqq Q(0)^{2}+c E_{0}^{1 / 2}\left(Q\left(t_{0}\right)+Q\left(t_{0}\right)^{3 / 2}\right) .
$$

Using the definition of $Q$ we obtain

$$
Q\left(t_{0}\right)^{2} \leqq Q(0)^{2}+c E_{0}^{1 / 2}\left(Q\left(t_{0}\right)+Q\left(t_{0}\right)^{3 / 2}\right),
$$


and by a trivial bootstrap argument we arrive at

Proposition 4.3. For any $(t, x)$ satisfying $|x| \geqq 1,|x|+1 \geqq \varepsilon t$ we have

$$
\begin{aligned}
& |\rho(t, x)| \leqq C|x|^{-2}, \\
& |\sigma(t, x)| \leqq C|x|^{-2}
\end{aligned}
$$

with some constant $C=c E_{0}^{1 / 4}+|F(0)|_{1}$ depending on the initial data but independent of $t, x$ and $\varepsilon$.

4.2. Estimate of the Component $\alpha$. To estimate $\alpha(t, x)$ for $1+|x| \geqq \varepsilon t$ consider the quantity

$$
R(t)=\sup _{\Sigma_{e}(t)}\left(|x|^{3 / 2}(1+\tau+|x|)|\alpha(\tau, x)|\right)
$$

Integrating now Eq. 4.10 along the characteristic $v=t+r=$ constant and repeating the argument used in the proof of Proposition 4.3 we obtain

$$
\begin{aligned}
\left|r_{0} \alpha\right|^{2} \leqq & \left|\left(t_{0}+\left|x_{0}\right|\right) \alpha\left(0, x_{0}+\frac{x_{0}}{\left|x_{0}\right|} t_{0}\right)\right|^{2} \\
& +c \int_{u_{*}}^{u_{0}} r^{2}|\alpha| \cdot(|D \rho|+|\not D \sigma|) d u
\end{aligned}
$$

where again $r_{0}=\left|x_{0}\right|, u_{0}=t_{0}-\left|x_{0}\right|, u_{*}=-t_{0}-\left|x_{0}\right|$ and $v_{0}=t_{0}+\left|x_{0}\right|$.

Applying the Cauchy-Schwarz inequality, estimate 4.16 and using the boundness of the conformal energy on backward light-cones (see Proposition 3.1), we find

$$
\begin{aligned}
\left|r_{0} \alpha\right|^{2} \leqq & C R(0)^{2}\left(1+t_{0}+\left|x_{0}\right|\right)^{-3} \\
& +C \int_{u_{*}}^{u_{0}} r|\alpha|(|\rho|+|\sigma|)\left(1+\left|r^{2} \sigma\right|^{1 / 2}\right) d u \\
\leqq & C R(0)^{2}\left(1+t_{0}+\left|x_{0}\right|\right)^{-2}\left|x_{0}\right|^{-1} \\
& +c E_{0}^{1 / 2}\left(1+C_{0}\right)^{1 / 2}\left(\int_{u_{*}}^{u_{0}}|\alpha|^{2}\left(1+v_{0}\right)^{-2} d u\right)^{1 / 2} \\
\leqq & C R(0)^{2}\left(1+t_{0}+\left|x_{0}\right|\right)^{-2}\left|x_{0}\right|^{-1} \\
& +C R\left(t_{0}\right)^{2}\left(1+v_{0}\right)^{-2} \cdot\left(\int_{\left|x_{0}\right|}^{t_{0}+\left|x_{0}\right|} r^{-3} d r\right)^{1 / 2} \\
\leqq & \left(R(0)^{2}+C R\left(t_{0}\right)\right)\left(1+t_{0}+\left|x_{0}\right|\right)^{-2}\left|x_{0}\right|^{-1} .
\end{aligned}
$$

Bootstrapping this inequality we obtain as before:

Proposition 4.4. For any $(t, x)$ satisfying $|x| \geqq 1,|x|+1 \geqq \varepsilon t$ we have

$$
|\alpha(t, x)| \leqq C\left(1+t_{0}+\left|x_{0}\right|\right)^{-1}\left|x_{0}\right|^{-3 / 2}
$$

with some constant $C=c E_{0}^{1 / 4}\left(1+c E_{0}^{1 / 2}|F(0)|_{1}\right)^{1 / 2}+|F(0)|_{1}$ depending on the initial data but independent of $t, x$ and $\varepsilon$. 
4.3. Estimate of the Component $\underline{\alpha}$. The estimates for the component $\underline{\alpha}$ are done somewhat differently, since we have only the possibility of integrating Eq. 4.9 along the ray $t-|x|=u_{0}=$ constant. The result is

$$
r_{0}^{2}\left|\underline{\alpha}\left(u_{0}, v_{0}\right)\right|^{2} \leqq\left|r_{*} \underline{\alpha}\right|^{2}\left(r_{*}, t_{*}\right)+c \int_{v_{*}}^{v_{0}} r^{2}|\underline{\alpha}|(|\not D \rho|+|D \sigma|) d v
$$

with $v_{*}=t_{*}+r_{*}$ and $t_{*}, r_{*}$ being determined by the equations

$$
\begin{aligned}
t_{*}-r_{*} & =u_{0}, \\
r_{*} & =-1+\varepsilon t_{*},
\end{aligned}
$$

if $u_{0} \geqq \sqrt{2}$ and

$$
\begin{aligned}
t_{*}-r_{*} & =u_{0}, \\
r_{*} & =1,
\end{aligned}
$$

if $0 \leqq u_{0} \leqq \sqrt{2}$. The first term in the right-hand side of Eq. 4.33 can be estimated as follows. Introduce the notation:

$$
\theta(t)=\sup _{\Sigma_{e}(t)}|x|(1+|\tau-| x||)|\underline{\alpha}(\tau, x)| .
$$

It follows that

$$
\left|r_{*} \underline{\alpha}\right|^{2}\left(r_{*}, t_{*}\right) \leqq c[F(t)]_{\infty}^{2}\left(1+t_{*}+r_{*}\right)^{-2},
$$

where $[F(t)]_{\infty}$ is the interior norm of $F$ appearing in Proposition 4.2. Now, recalling that $\left(1+t_{*}+r_{*}\right) \geqq 1+\left|t_{*}-r_{*}\right|=1+u_{0}$, we have

$$
\begin{aligned}
r_{0}^{2}\left|\underline{\alpha}\left(u_{0}, v_{0}\right)\right|^{2} & \leqq c[F(t)]_{\infty}^{2}\left(1+\left|t_{0}-r_{0}\right|\right)^{-2}+c\left(\int_{v_{*}}^{v_{0}} r|\underline{\alpha}|(|\rho|+|\sigma|)\left(1+\left|r^{2} \sigma\right|^{1 / 2} d v\right)\right. \\
& \leqq c[F(t)]_{\infty}^{2}\left(1+\left|t_{0}-r_{0}\right|\right)^{-2}+c\left(\int_{v_{*}}^{v_{0}} r|\underline{\alpha}|(|\rho|+|\sigma| d v) .\right.
\end{aligned}
$$

In the last inequality we used the estimates of the component $\sigma$ which have been already proved in Proposition 4.3. Now, applying Proposition 3.1 to the advanced cone $K_{p}$ with vertex at $p=\left(-u_{0}, 0,0,0\right)$ we can bound one of the factors in the last integral by the conformal energy of $F$ and obtain the estimate

$$
\begin{aligned}
r_{0}^{2}\left|\underline{\alpha}\left(u_{0}, v_{0}\right)\right|^{2} \leqq & c[F(t)]_{\infty}^{2}\left(1+\left|t_{0}-r_{0}\right|\right)^{-2} \\
& +c E_{0}^{1 / 2}\left(1+C_{0}\right)^{1 / 2}\left(\int_{v_{*}}^{v_{0}}|\underline{\alpha}|^{2}(1+u)^{-2} d v\right)^{1 / 2} .
\end{aligned}
$$

Multiplying 4.36 through by the appropriate weights and using the definition of $\theta$ we get

$$
\begin{aligned}
\theta\left(t_{0}\right)^{2} & \leqq c[F(t)]_{\infty}^{2}+C \theta\left(t_{0}\right)\left(\int_{v_{*}}^{v_{0}} r^{-2} d v\right)^{1 / 2} \\
& \leqq c[F(t)]_{\infty}^{2}+C \theta\left(t_{0}\right)
\end{aligned}
$$


Bootstrapping the last inequality we arrive at:

$$
|x|(1+|t-| x||)|\underline{\alpha}(t, x)| \leqq c[F(t)]_{\infty}
$$

for all $x$ and $t$ in the exterior region such that $|x| \leqq t$. The case when $|x| \geqq t$ is analogous and we get

$$
|x|(1+|t-| x||)|\alpha(t, x)| \leqq C .
$$

Combining estimates 4.37 and 4.38 we arrive at:

Proposition 4.5. For any $(t, x)$ satisfying $|x| \geqq 1,1+|x| \geqq \varepsilon t$ we have

$$
|\underline{\alpha}(t, x)| \leqq C|x|^{-1}(1+|t-| x||)^{-1}\left(1+[F(t)]_{\infty}\right)
$$

with some constant $C=c E_{0}^{1 / 4}\left(1+c E_{0}^{1 / 2}|F(0)|_{1}\right)^{1 / 2}+|F(0)|_{1}$ depending on the initial data but independent of $t, x$ and $\varepsilon$.

\section{Estimates of the Solution for $|x|+1 \leqq \varepsilon t$}

In this section we shall estimate the curvature tensor $F$ in a neighborhood of the central line $r=0$, namely on the region where

$$
|x|+1 \leqq \varepsilon t \quad \text { or } \quad|x| \leqq 1 .
$$

Here $\varepsilon$ is a fixed positive real number depending only on the initial data. If $0 \leqq t_{0} \leqq \frac{2}{\varepsilon}$, then the approach used in [4] shows that the $L^{\infty}$-norm of the curvature tensor $F\left(t_{0}, \cdot\right)$ is bounded. Therefore it is only necessary to consider the case when $t_{0} \geqq \frac{2}{\varepsilon}$, where $\varepsilon$ is a fixed number to be specified later. The basic idea in the subsequent estimates consists of using a wave equation for $F$. By straightforward differentiation we get from Eqs. 1.1-1.2:

$$
\square_{A} F_{\alpha \beta}=2\left[F_{\alpha}^{\gamma}, F_{\gamma \beta}\right],
$$

where $\square_{A}=-D_{\lambda} D^{\lambda}$ denotes the wave operator relative to the Yang-Mills potential $A$. Writing 5.1 explicitly in terms of the gauge potential, we find

$$
\begin{aligned}
\square F_{\alpha \beta}= & -2 \partial_{\gamma}\left(\left[A^{\gamma}, F_{\alpha \beta}\right]\right)+\left[\partial_{\gamma} A_{\gamma}, F_{\alpha \beta}\right] \\
& -\left[A^{\gamma},\left[A_{\gamma}, F_{\alpha \beta}\right]\right]+2\left[F_{\alpha}^{\gamma}, F_{\gamma \beta}\right] .
\end{aligned}
$$

Let us consider now a fixed point $p=\left(t_{0}, x_{0}\right)$ with $\left|x_{0}\right|+1 \leqq \varepsilon t_{0}$ and $t_{0} \geqq \frac{2}{\varepsilon}$. Following again [4] we use a Cronström gauge adapted to the point $p$, that is to say:

$$
\left(t-t_{0}\right) A_{0}(t, x)+\sum_{j=1}^{3}\left(x^{j}-x_{0}^{j}\right) A_{j}(t, x)=0 .
$$


Using the fundamental solution representation of the wave equation we may write (cf. [4]):

$$
\begin{aligned}
F_{\alpha \beta}(p)= & F_{\alpha \beta}^{L I N}(p)-\frac{1}{4 \pi} \int_{K_{p}} r d r d \omega\left(-2 \partial_{\gamma}\left(\left[A^{\gamma}, F_{\alpha \beta}\right]\right)\right. \\
& \left.+\left[\partial_{\gamma} A_{\gamma}, F_{\alpha \beta}\right]-\left[A^{\gamma},\left[A_{\gamma}, F_{\alpha \beta}\right]\right]+2\left[F_{\alpha}^{\gamma}, F_{\gamma \beta}\right]\right) \\
:= & F_{\alpha \beta}^{L I N}(p)-\frac{1}{4 \pi}\left(I_{1}+I_{2}+I_{3}+I_{4}\right),
\end{aligned}
$$

where $F_{\alpha \beta}^{L I N}(p)$ is the solution of the wave equation $\square F_{\alpha \beta}^{L I N}=0$ with the same initial data as $F_{\alpha \beta}$, and $I_{1}, \ldots, I_{4}$ denote the terms containing the non-linearities. The bound on $F_{\alpha \beta}^{L I N}$ is well-known: ${ }^{7}$

$$
\left|F_{\alpha \beta}^{L I N}(p)\right| \leqq C\left(1+t_{0}\right)^{-5 / 2}
$$

for every $\left|x_{0}\right|+1 \leqq \varepsilon t_{0}$ with $C$ depending on the norm 1.8 .

In order to estimate the non-linear terms $I_{1}, I_{2}, I_{3}, I_{4}$ in the right-hand side of Eq. 5.4 we shall use the local null frame 2.6-2.7.

We will proceed now with the estimates of the non-linear terms in Eq. 5.4. Let us estimate $I_{1}$. Here one is better off if we translate the coordinates of the point $p$ to the origin $(0,0)$. Integrating in the angular variables:

$$
\begin{aligned}
I_{1}\left(t_{0}, x_{0}\right) & =\int_{0}^{t_{0}} \int_{S^{2}} r d r d \omega\left(\partial_{\mu}\left[A^{\mu}, F_{\alpha \beta}\right]\right)(-r, r \omega) \\
& =\int_{0}^{t_{0}} \int_{S^{2}} r d r d \omega\left(\partial_{3} P^{3}+\partial_{4} P^{4}\right)(-r, r \omega),
\end{aligned}
$$

where $P^{\mu}=\left[A^{\mu}, F_{\alpha \beta}\right]$. Recalling that the gauge condition 5.3 implies $x_{\mu} P^{\mu}=0$, we obtain by differentiation with respect to $x^{4}$ :

$$
P_{4}+x_{\mu} \partial_{4} P^{\mu}=0 \text {. }
$$

Since on $K_{p}$ we have ${ }^{(p)} x^{4}=0$, then one obtains

$$
P_{4}+r \partial_{4} P^{4}=0
$$

and then

$$
\begin{aligned}
I_{1}\left(t_{0}, x_{0}\right) & =-\int_{0}^{t_{0}} \int_{S^{2}} d r d \omega \partial_{r}\left(r P_{4}\right) \\
& =-\int_{S^{2}} d \omega P_{4}\left(0, x_{0}-t_{0} \omega\right) .
\end{aligned}
$$

Using the fact that $|P(0, x)| \leqq|A(0, x)| \cdot|F(0, x)|$ and the decay of the initial data, we get $^{8}$

$$
|P(0, x)| \leqq c(1+|x|)^{-7 / 2}
$$

\footnotetext{
${ }^{7}$ See $[10]$ for example

${ }^{8}$ Observe that in the class of canonical gauges the potential $A$ cannot be made to decay faster than $r^{-1}$
} 
with $x=x_{0}-t_{0} \omega$. Now, if $\left|x_{0}\right| \leqq \frac{t_{0}}{5}$, then

$$
|x| \geqq t_{0}-\left|x_{0}\right| \geqq \frac{4}{5} t_{0}
$$

and then

$$
\left|I_{1}\left(t_{0}, x_{0}\right)\right| \leqq c\left(1+t_{0}\right)^{-5 / 2}
$$

for all points in the interior region, provided we choose $\varepsilon$ such that $\varepsilon<1 / 5$.

The integrals $I_{2}$ and $I_{3}$ are estimated similarly. It follows from the local gauge 5.3 that on the cone $K_{p}$ we can represent the potentials $A$ in terms of the curvature as

$$
\begin{aligned}
A_{v}(x) & =\int_{0}^{1}\left({ }^{(p)} x^{3} F_{3 v}(p+\lambda(x-p))\right) \lambda d \lambda \\
& =\int_{0}^{1} r F_{3 v}\left(t_{0}-\lambda r, x_{0}+\lambda r \omega\right) \lambda d \lambda, \\
\partial^{v} A_{v}(x) & =\int_{0}^{1}{ }^{(p)} x^{3}\left[F_{3 v}(p+\lambda(x-p)), A^{v}(p+\lambda(x-p)) \lambda^{2} d \lambda\right. \\
& =\int_{0}^{1} r\left[F_{3 v}, A^{v}\right]\left(t_{0}-\lambda r, x_{0}+\lambda r \omega\right) \lambda^{2} d \lambda
\end{aligned}
$$

for all $x$ in the cone $K_{p}$ (see [4]). Inserting these formulas in the integrals $I_{2}$ and $I_{3}$ we obtain

$$
\begin{aligned}
I_{2}= & \int_{0}^{t_{0}} \int_{S^{2}} r^{3} d r d \omega \int_{0}^{1} \lambda d \lambda \int_{0}^{1} \mu d \mu\left[\left[F _ { 3 A } \left(\left(t_{0}-\lambda r, x_{0}+\lambda r \omega\right),\right.\right.\right. \\
& \left.\left.F_{3}^{A}\left(t_{0}-\mu r, x_{0}+\mu r \omega\right)\right], F_{\alpha \beta}\left(t_{0}-r, x_{0}+r \omega\right)\right], \\
I_{3}= & \int_{0}^{t_{0}} \int_{S^{2}} r^{3} d r d \omega \int_{0}^{1} \lambda d \lambda \int_{0}^{\lambda} \mu d \mu\left[\left[F _ { 3 A } \left(\left(t_{0}-\lambda r, x_{0}+\lambda r \omega\right),\right.\right.\right. \\
& \left.\left.F_{3}^{A}\left(t_{0}-\mu r, x_{0}+\mu r \omega\right)\right], F_{\alpha \beta}\left(t_{0}-r, x_{0}+r \omega\right)\right],
\end{aligned}
$$

and from the Cauchy-Schwarz inequality:

$$
\begin{aligned}
\left|I_{2}\right|+\left|I_{3}\right| \leqq & c \int_{0}^{t_{0}} r^{3} d r \sup _{\omega}\left|F\left(t_{0}-r, x_{0}+r \omega\right)\right| \\
& \cdot\left(\int_{0}^{1} \lambda d \lambda\left(\int_{S^{2}} d \omega \mid \underline{\alpha}\left(\left.\left(t_{0}-\lambda r, x_{0}+\lambda r \omega\right)\right|^{2}\right)^{1 / 2}\right)^{2} .\right.
\end{aligned}
$$

We appeal now to the following lemma:

Lemma 5.1. If $0<a<1$, then:

$$
\left(\int_{0}^{1} \lambda f(\lambda) d \lambda\right)^{2} \leqq 2 a \int_{0}^{a}|f(\lambda)|^{2} \lambda^{2} d \lambda+\frac{2}{a} \int_{a}^{1}|f(\lambda)|^{2} \lambda^{4} d \lambda .
$$


Proof of the lemma. Starting from

$$
\left(\int_{0}^{1} \lambda f(\lambda) d \lambda\right)^{2} \leqq 2\left(\int_{0}^{a} \lambda f(\lambda) d \lambda\right)^{2}+2\left(\int_{a}^{1} \lambda f(\lambda) d \lambda\right)^{2}
$$

and using the Cauchy-Schwarz inequality

$$
\left(\int_{0}^{a} \lambda f(\lambda) d \lambda\right)^{2} \leqq \int_{0}^{a} 1 d \lambda \int_{0}^{a} \lambda^{2}|f(\lambda)|^{2} d \lambda=a \int_{0}^{a} \lambda^{2}|f(\lambda)|^{2} d \lambda
$$

Similarly

$$
\left(\int_{0}^{1} \lambda f(\lambda) d \lambda\right)^{2} \leqq\left(\int_{a}^{1} \frac{d \lambda}{\lambda^{2}}\right) \cdot\left(\int_{a}^{1}|f(\lambda)|^{2} \lambda^{4} d \lambda\right) \leqq \frac{1}{a} \int_{a}^{1}|f(\lambda)|^{2} \lambda^{4} d \lambda
$$

This completes the proof.

We proceed now by dividing the region of integration in the right-hand side of (5.7) into three parts and applying the previous lemma with $a=1 / 5$. The result is

$$
\begin{aligned}
& \left|I_{2}\right|+\left|I_{3}\right| \leqq c \int_{0}^{t_{0} / 5} r^{3} d r \sup _{\omega}\left|F\left(t_{0}-r, x_{0}+r \omega\right)\right| \int_{0}^{1} \lambda^{2} d \lambda \int_{S^{2}} d \omega\left|\underline{\alpha}\left(t_{0}-\lambda r, x_{0}+\lambda r \omega\right)\right|^{2} \\
& +c \int_{t_{0} / 5}^{t_{0}} r^{3} d r \sup _{\omega}\left|F\left(t_{0}-r, x_{0}+r \omega\right)\right| \int_{0}^{1 / 5} \lambda^{2} d \lambda \int_{S^{2}} d \omega\left|\underline{\alpha}\left(t_{0}-\lambda r, x_{0}+\lambda r \omega\right)\right|^{2} \\
& +c \int_{t_{0} / 5}^{t_{0}} r^{3} d r \sup _{\omega}\left|F\left(t_{0}-r, x_{0}+r \omega\right)\right| \int_{1 / 5}^{1} \lambda^{4} d \lambda \int_{S^{2}} d \omega\left|\underline{\alpha}\left(t_{0}-\lambda r, x_{0}+\lambda r \omega\right)\right|^{2} .
\end{aligned}
$$

The first of these terms can be estimated as follows. Making the change of variables $\bar{r}=\lambda r$ we obtain

$$
r^{3} \int_{0}^{1} \lambda^{2} d \lambda \int_{S^{2}} d \omega\left|\underline{\alpha}\left(t_{0}-\lambda r, x_{0}+\lambda r \omega\right)\right|^{2}=\int_{0}^{r} \bar{r}^{2} d \bar{r} \int_{S^{2}} d \omega\left|\underline{\alpha}\left(t_{0}-\bar{r}, x_{0}+\bar{r} \omega\right)\right|^{2} .
$$

Now we remark that for $y=x_{0}+\bar{r} \omega$ with $|\bar{r}| \leqq t_{0} / 5,\left|x_{0}\right| \leqq t_{0} / 5$ we have

$$
|y| \leqq \frac{2}{5} t_{0} \leqq\left(t_{0}-r\right) / 2,
$$

and then

$$
\begin{aligned}
\int_{0}^{r} \bar{r}^{2} d \bar{r} \int_{S^{2}} d \omega\left|\underline{\alpha}\left(t_{0}-\bar{r}, x_{0}+\bar{r} \omega\right)\right|^{2} & \leqq \int_{|y| \leqq \frac{1}{2}\left|t_{0}-r\right|}\left|\underline{\alpha}\left(t_{0}-r, y\right)\right|^{2} d y \\
& \leqq c E_{0} t_{0}^{-2},
\end{aligned}
$$

so that we obtain the estimate

$$
\begin{gathered}
\int_{0}^{t_{0} / 5} r^{3} d r \sup _{\omega}\left|F\left(t_{0}-r, x_{0}+r \omega\right)\right| \int_{0}^{1} \lambda^{2} d \lambda \int_{S^{2}} d \omega\left|\underline{\alpha}\left(t_{0}-\lambda r, x_{0}+\lambda r \omega\right)\right|^{2} \\
\leqq c E_{0} t_{0}^{-2} \int_{0}^{t_{0} / 5} d r \sup _{\omega}\left|F\left(t_{0}-r, x_{0}+r \omega\right)\right|
\end{gathered}
$$


To estimate this last integral one must recall the definition of the interior norm 4.21 and observe that for the point $t=t_{0}-r, x=x_{0}+r \omega$, with $\left|x_{0}\right| \leqq t_{0} / 2, t_{0} \geqq 1$, we have:

$$
t+|x|=t_{0}-r+\left|x_{0}+r \omega\right| \geqq t_{0}-r+r-\left|x_{0}\right|=t_{0}-x_{0} \geqq c\left(1+t_{0}\right),
$$

so that

$$
\sup _{\omega}\left|F\left(t_{0}-r, x_{0}+r \omega\right)\right| \leqq \frac{c\left(1+\left[F\left(t_{0}\right)\right]_{\infty}\right)}{\left(1+t_{0}\right)} .
$$

We have made use here of the estimates of Sect. 4 valid for points in the exterior region $1+|x| \geqq \varepsilon t$.

Remarking now that for $\left|x_{0}\right|+1 \leqq \varepsilon t_{0}$ and $t_{0} \geqq 1$ one has

$$
\frac{1}{t_{0}^{2}} \leqq \frac{C \varepsilon}{\left(1+\left|x_{0}\right|\right)\left(1+t_{0}+\left|x_{0}\right|\right)} .
$$

We obtain the final estimate

$$
\begin{aligned}
\int_{0}^{t_{0} / 5} r^{3} d r \sup _{\omega}\left|F\left(t_{0}-r, x_{0}+r \omega\right)\right| \int_{0}^{1} \lambda^{2} d \lambda & \int_{S^{2}} d \omega\left|\underline{\alpha}\left(t_{0}-\lambda r, x_{0}+\lambda r \omega\right)\right|^{2} \\
& \leqq \frac{C \varepsilon\left(1+\left[F\left(t_{0}\right)\right]_{\infty}\right)}{\left(1+\left|x_{0}\right|\right)\left(1+t_{0}+\left|x_{0}\right|\right)}
\end{aligned}
$$

with $C$ independent of $\varepsilon$.

The second integral is estimated in a similar fashion and we obtain:

$$
\begin{gathered}
\int_{t_{0} / 5}^{t_{0}} r^{3} d r \sup _{\omega}\left|F\left(t_{0}-r, x_{0}+r \omega\right)\right| \int_{0}^{1 / 5} \lambda^{2} d \lambda \int_{S^{2}} d \omega\left|\underline{\alpha}\left(t_{0}-\lambda r, x_{0}+\lambda r \omega\right)\right|^{2} \\
\leqq \frac{C \varepsilon\left(1+\left[F\left(t_{0}\right)\right]_{\infty}\right)}{\left(1+\left|x_{0}\right|\right)\left(1+t_{0}+\left|x_{0}\right|\right)} .
\end{gathered}
$$

The third term is estimated directly in terms of the conformal energy

$$
r^{3} \int_{0}^{1} \lambda^{2} d \lambda \int_{S^{2}} d \omega\left|\underline{\alpha}\left(t_{0}-\lambda r, x_{0}+\lambda r \omega\right)\right|^{2} \leqq E_{0}<+\infty .
$$

Remarking that for $r_{0} \geqq t_{0} / 5$ and $\varepsilon<1 / 5$ one has the inequality

$$
\left|x_{0}+r \omega\right| \geqq r-\left|x_{0}\right| \geqq(1 / 5-\varepsilon) t_{0} \geqq c t_{0} .
$$

One finally concludes that

$$
\left|I_{2}\right|+\left|I_{3}\right| \leqq \frac{C \varepsilon\left(1+\left[F\left(t_{0}\right)\right]_{\infty}\right)}{\left(1+\left|x_{0}\right|\right)\left(1+t_{0}+\left|x_{0}\right|\right)} .
$$

Finally, let us estimate the term

$$
I_{4}\left(t_{0}, x_{0}\right)=\int_{0}^{t_{0}} \int_{S^{2}} r d r d \omega\left[F_{\alpha \mu}, F_{\beta}^{\mu}\right]\left(t_{0}-r, x_{0}+r \omega\right) .
$$


We decompose the cone $K_{p}$ into two pieces:

$$
\begin{aligned}
& K_{p}^{+}=K_{p} \cap\{t / 2 \leqq|x| \leqq 2 t\}, \\
& K_{p}^{-}=K_{p}-K_{p}^{+},
\end{aligned}
$$

and denote

$$
I_{4}^{ \pm}\left(t_{0}, x_{0}\right)=\int_{K_{p}^{ \pm}} r d r d \omega\left[F_{\alpha \mu}, F_{\beta}^{\mu}\right]\left(t_{0}-r, x_{0}+r \omega\right) .
$$

These two integrals consist of quadratic terms which are gauge-covariant. Both integrals are estimated by examining the special structure of the terms that appear in the commutator. This plays the role here of the null condition of Klainerman ([9]). The lower part of the integral is estimated by using once again the local null frame (2.5) whereas the upper part is estimated by using a global null frame relative to the origin.

Let us estimate $I_{4}^{-}$. In the local null frame we can express the term $\left[F_{\alpha \mu}, F_{\beta}^{\mu}\right]$ as a linear combination of terms

$$
\left[{ }^{(p)} F_{\sigma v},{ }^{(p)} F_{\xi}^{v}\right]
$$

where $\sigma, v$ and $\xi$ denote the coordinates in the local frame. The important thing to notice is that the coefficients of this linear combinations are all uniformly bounded (cf. [4])

$$
\left|\left[F_{\alpha \mu}, F_{\beta}^{\mu}\right] \leqq C \sup _{\sigma, \xi}\right|\left[{ }^{(p)} F_{\sigma \nu},{ }^{(p)} F_{\xi}^{\nu}\right] \mid
$$

Now, for different choices of the null indices, the right-hand side of (5.13) will have the form

$$
\begin{aligned}
& {\left[{ }^{(p)} F_{3 A},{ }^{(p)} F_{4}^{A}\right]=[\underline{\alpha}, \alpha],} \\
& {\left[{ }^{(p)} F_{3 B},{ }^{(p)} F_{A}^{B}\right]=[\underline{\alpha}, \sigma],} \\
& {\left[{ }^{(p)} F_{34},{ }^{(p)} F_{A}^{4}\right]=[\rho, \underline{\alpha}],} \\
& {\left[{ }^{(p)} F_{4 B},{ }^{(p)} F_{A}^{B}\right]=[\alpha, \sigma],} \\
& {\left[{ }^{(p)} F_{43},{ }^{(p)} F_{A}^{3}\right]=[\rho, \alpha],}
\end{aligned}
$$

and zero for all other choices of $\sigma$, $\xi$. Setting now

$$
\begin{aligned}
F_{+} & =(\alpha, \rho, \sigma), & F_{-}=(\underline{\alpha}, \rho, \sigma), \\
\left|F_{+}\right| & =|\alpha|+|\rho|+|\sigma|, & \left|F_{-}\right|=|\underline{\alpha}|+|\rho|+|\sigma| .
\end{aligned}
$$

Then each of the terms in (5.13) satisfies the estimate

$$
\left[{ }^{(p)} F_{\sigma v},{ }^{(p)} F_{\xi}^{\nu}\right] \leqq c\left|F_{+}\right| \cdot\left|F_{-}\right|,
$$

and then we obtain

$$
\left|I_{4}^{-}\right| \leqq c \int_{K_{\bar{p}}^{-}}\left|F_{+}\right| \cdot\left|F_{-}\right| r d r d \omega
$$


We remark now that $F_{-}$contains exactly the components which can be absorbed in the conformal energy in $K_{p}$, so that at least one of the terms can be taken in the $L^{2}$-norm. Using the boundness of the conformal energy, we get

$$
\begin{aligned}
\left|I_{4}^{-}\right| & \leqq c\left(\int_{K_{\bar{p}}} r^{2} \tau_{-}^{2}\left|F_{-}\right|^{2} d r d \omega\right)^{1 / 2} \cdot\left(\int_{K_{\bar{p}}} \tau_{-}^{-2}\left|F_{+}\right|^{2} d r d \omega\right)^{1 / 2} \\
& \leqq c E_{0}^{1 / 2}\left(\int_{K_{\bar{p}}^{-}} \tau_{-}^{-2}\left|F_{+}\right|^{2} d r d \omega\right)^{1 / 2} .
\end{aligned}
$$

The important thing to notice now is that on the part $K_{p}^{-}$of the light-cone $K_{p}$ the retarded time $\tau_{-}$is equivalent to the advanced time $\tau_{+}$so that

$$
\tau_{-}=1+\left|t_{0}-r-\right| x_{0}+r \omega|| \geqq C\left(1+t_{0}\right)
$$

by using (5.8). Here $\left|F_{+}\right|$is the component relative to the local decomposition of $F$. It can be estimated by the invariant norm of $F$ which by its turn can be estimated as in (5.9) by the exterior norms (cf. estimate 4.2 of Sect. 4). We obtain this way

$$
\begin{aligned}
\left|I_{4}^{-}\right| & \leqq c\left(\int_{S^{2}}^{t_{0}} \frac{1}{1+\left|t_{0}-r\right|^{2}} d r d \omega\right)^{1 / 2} \cdot \frac{\left(1+\left[F\left(t_{0}\right)\right]_{\infty}\right)}{t_{0}^{2}} \\
& \leqq \frac{C \varepsilon\left(1+\left[F\left(t_{0}\right)\right]_{\infty}\right)}{\left(1+\left|x_{0}\right|\right)\left(1+t_{0}+\left|x_{0}\right|\right)} .
\end{aligned}
$$

In the last step we have used the fact that $\left|x_{0}\right|+1 \leqq \varepsilon t_{0}$.

To obtain a similar estimate for $I_{4}^{+}$we use instead a null frame with respect the origin and repeat the same arguments that led to inequality (5.14). The main thing to remark is that if $\varepsilon$ is sufficiently small (say $\varepsilon<1 / 2$ ), then we have the inclusion $K_{p}^{+} \subset K_{p} \cap\{|x|+1 \geqq \varepsilon t\}$. In particular, we are in position to apply the estimates from Sect. 4 and obtain

$$
\begin{aligned}
& \left|F_{+}(t, x)\right| \leqq \frac{C}{(1+t+|x|)^{2}} \\
& \left|F_{-}(t, x)\right| \leqq \frac{C\left(1+[F(t)]_{\infty}\right)}{(1+|t-| x||)(1+t+|x|)}
\end{aligned}
$$

for all $(t, x) \in K_{p}^{+}$. By restricting further the choice of $\varepsilon(\varepsilon<3 / 8$ say), one can show that for points $(t, x)$ in $K_{p}^{+}$, one has $r \geqq t_{0} / 2$ and we arrive therefore at

$$
\left|I_{4}^{+}\right| \leqq C \int_{S^{2}} \int_{t_{0} / 2}^{t_{0}} \frac{1}{1+\left|t_{0}-r-\right| x_{0}+r \omega||} d r d \omega \cdot \frac{\left(1+\left[F\left(t_{0}\right)\right]_{\infty}\right)}{t_{0}^{2}} .
$$

The last integral can be estimated by using a lemma due to F. John (see [10] also) which exploits an integration over the angular variables:

$$
\begin{aligned}
\int_{S^{2}} \int_{t_{0} / 2}^{t_{0}} \frac{1}{1+\left|t_{0}-r-\right| x_{0}+r \omega||} d r d \omega & =\int_{t_{0} / 2}^{t_{0}} \frac{1}{r^{2}}\left(\int_{\left|y-x_{0}\right|=r} \frac{d \omega_{y}}{1+\left|t_{0}-r-\right| y||}\right) d r \\
& =\int_{t_{0} / 2}^{t_{0}} \frac{2 \pi}{r\left|x_{0}\right|}\left(\int_{\left|x_{0}\right|-r \mid}^{\left|x_{0}\right|+r} \frac{\lambda d \lambda}{1+\left|t_{0}-r-\lambda\right|}\right) d r . \\
& =C_{0}
\end{aligned}
$$


The range of integration in the variable $r$ avoids the logarithmic contribution coming from the integral in $\lambda$. The constant $C_{0}$ is independent of the point $\left(t_{0}, x_{0}\right)$. We obtain then

$$
\left|I_{4}^{+}\right| \leqq \frac{C \varepsilon\left(1+\left[F\left(t_{0}\right)\right]_{\infty}\right)}{\left(1+\left|x_{0}\right|\right)\left(1+t_{0}+\left|x_{0}\right|\right)} .
$$

Combining now (5.6), (5.12), (5.14) and (5.15), we have

$$
\left|F\left(t_{0}, x_{0}\right)\right| \leqq \frac{C \varepsilon}{\left(1+\left|x_{0}\right|\right)\left(1+t_{0}+\left|x_{0}\right|\right)}\left(1+\left[F\left(t_{0}\right)\right]_{\infty}\right)
$$

for all $\left|x_{0}\right|+1 \leqq \varepsilon t_{0}$ and $t_{0} \geqq 2 / \varepsilon$. Choosing now $\varepsilon$ sufficiently small that $C \varepsilon<1 / 2$ we can bootstrap the last inequality and finally arrive at

$$
\left[F\left(t_{0}\right)\right]_{\infty} \leqq C,
$$

which was the desired goal. This completes the estimate of the curvature tensor near the central line. Combining (5.16) with the estimate (4.17-4.20) from Sect. 4, we conclude the proof of the main theorem.

Acknowledgements. One of the authors (V.G.) would like to thank the hospitality of the Institut für Angewandte Mathematik in Bonn, where part of this work was accomplished. We also would like to thank Sergiu Klainerman for his criticisms on the first version of the manuscript.

\section{References}

1. Choquet-Bruhat, Y., Paneitz, S., Segal, I.: The Yang-Mills equations on the universal cosmos. J. Funct. Anal. 53, 112 (1983)

2. Choquet-Bruhat, Y.: Lectures on global solutions of hyperbolic equations of gauge theories. Proceedings of Symposium at Simon Bolivar University, Caracas, Aragone, C. (ed.)

3. Christodoulou, D.: Solutions globales des equations de champs de Yang-Mills. C.R. Acad. Sci. Paris 293, Ser. A, 39 (1981)

4. Eardley, D., Moncrief, V.: The global existence of Yang-Mills-Higgs fields in 4-dimensional Minkowski space. Commun. Math. Phys. 83, 171 (1982)

5. Georgiev, V.: Small-amplitude solutions of the Maxwell-Dirac equations. Indiana Univ. Math. J. 40(3), 845 (1991)

6. Glassey, R.T., Strauss, W.A.: The Scattering of Yang-Mills fields. Commun. Math. Phys. 89, 465 (1983)

7. Hawking, S., Ellis, G.: The large scale structure of space-time. Cambridge: Cambridge University Press 1978

8. Jaffe, A., Taubes, C.: Vortices and monopoles. Birkhäuser Progress in Physics PPh2, New York, Basel: Birkhäuser 1980

9. Klainerman, S.: The null condition and global existence to non-linear wave equations. Lectures in Applied Mathematics, Vol. 23, Nicolaenko, B. (ed.), 1986

10. Pecher, H.: Scattering for semilinear wave equations with small data in three space dimensions. Math. Zeit. 198, 77 (1988)

11. Schirmer, P.P.: Global existence for spherically symmetric Yang-Mills fields on $3+1$ spacetime dimensions. Doctoral dissertation, New York University, 1990

12. Schirmer, P.P.: Decay estimates for spherically symmetric Yang-Mills fields in Minkowski space. Ann. Inst. Henri Poincaré, to appear

13. Shu, W.T.: Commun. Math. Phys. 140, 449-480 (1991) 